\title{
Sporadic Examples of Directed Strongly Regular Graphs Obtained by Computer Algebra Experimentation (Extended version)
}

\author{
Štefan Gyürki \\ Matej Bel University, Faculty of Natural Sciences \\ Tajovského 40, 97401 Banská Bystrica, Slovak Republic, e-mail: gypista@gmail.com \\ Mikhail Klin \\ Department of Mathematics, Ben-Gurion University of the Negev \\ 84105 Beer Sheva, Israel, e-mail: klin@cs.bgu.ac.il
}

June 7, 2021

\begin{abstract}
We report about the results of the application of modern computer algebra tools for construction of directed strongly regular graphs. The suggested techniques are based on the investigation of non-commutative association schemes and Cayley graphs over non-Abelian groups. We demonstrate examples of directed strongly regular graphs for 28 different parameter sets, for which the existence of a corresponding digraph has not been known before.
\end{abstract}

\section{Introduction}

This project is devoted to the computer algebra experimentation in the area of algebraic graph theory, the part of mathematics on the edge between graph theory, linear algebra, and group theory. The main objects of interest in algebraic graph theory are highly symmetric graphs, where level of symmetry might be measured both on group-theoretical and purely combinatorial levels. Two books [1] and [1] reflect impressive progress in this part of mathematics.

Nowadays computer algebra tools, and especially GAP (Groups, Algorithms, Programming - a System for Computational Discrete Algebra [9]), together with a few of its share packages, become an inalienable part of modern graph theory and combinatorics. A significant portion of striking combinatorial structures was discovered and analyzed with the aid of a computer. The main subject of interest in the presented text are directed strongly regular graphs (briefly DSRGs), a natural generalization of a classical (in 
algebraic graph theory) concept from simple to directed graphs. The concept of a DSRG was suggested and investigated by A. Duval in [5. For a while it remained unnoticed, however, during last 15 years this class of structures is becoming more and more popular.

The initial concept of a strongly regular graph (briefly SRG) has a number of relatively independent origins of interest in such diverse areas like design of statistical experiments, finite geometries, applied permutation groups, and also complexity theory of algorithms. Indeed, it is well-known that SRGs are usually regarded as most sophisticated structures for the problems of isomorphism testing of graphs and determination of the automorphism group of graphs. The main combinatorial invariant of a SRG is its parameter set, in the sense of 2. Typically, classification of SRGs is arranged for each parameter set separately. Similar situation is also observed for DSRGs. However, these structures appear even more frequently. For example, while there are 36 parameter sets for SRGs on up to 50 vertices, this number is 225 for DSRGs. On the other hand, the central problems of the identification of DSRGs and determination of their symmetry are on the same level of difficulty as it appears for the classical case of strongly regular graphs.

In this context, DSRGs provide, in comparison with SRGs much more wide training polygons for the experts in the complexity theory which allows more diversity (undirected versus directed) for investigated graphs.

The previous experience (earned, in particular, by M. Klin and his coauthors) shows that a clever use of computers helps to discover new examples of DSRGs and after that to reach an honest theoretical generalization of the detected structures. This line of activity stimulated the authors to join their efforts in a new attempt. At this stage, we are concentrating on the association schemes as possible origins of new DSRGs. Namely, we wish to consider any association scheme $\mathcal{M}$, for which a suitable union of classes provides a DSRG, preferably new, moreover, with a new parameter set.

This text is an extended version of a paper (with the same title) to appear soon in a special volume (CASC 2014) of LNCS series.

The paper is organized as follows. In Section 2, the necessary basic notions are introduced. In Section 3, we describe our approach to the problem of finding new directed strongly regular graphs using computer algebra experimentation. In Section 4, the mentioned strategies are explained with enough rigorous details and the results of different approaches are reported. In Section 5, the results of a classical strategy using Cayley graphs are submitted. We conclude with a discussion and summary of new graphs, being discovered. Information, provided in the Appendix, allows one to reconstruct (with the aid of a computer) all new DSRGs discovered by us.

\section{Preliminaries}

Below we present brief account of most significant concepts exploited in the paper. We refer to [2] and [19] for more information.

\subsection{General Concepts}

A simple graph $\Gamma$ is a pair $(V, E)$, where $V$ is a finite set of vertices, and $E$ is a set of 2-subsets of $V$ which are called edges. 
A directed graph (briefly digraph) $\Gamma$ is a pair $(V, R)$ where $V$ is the set of vertices and $R$ is a binary relation on $V$, that is a subset of the set $V^{2}$ of all ordered pairs of elements in $V$. The pairs in $R$ are called directed arcs or darts. The vertex set of $\Gamma$ is denoted by $V(\Gamma)$ and the dart set is denoted by $R(\Gamma)$.

A balanced incomplete block design (BIBD) is a pair $(\mathcal{P}, \mathcal{B})$ where $\mathcal{P}$ is the point set of cardinality $v$, and $\mathcal{B}$ is a collection of $b k$-subsets of $\mathcal{P}$ (blocks) such that each element of $\mathcal{P}$ is contained in exactly $r$ blocks and any 2-subset of $V$ is contained in exactly $\lambda$ blocks. The numbers $v, b, r, k$, and $\lambda$ are parameters of the BIBD. From the parameters $v, k, \lambda$ the remaining two are determined uniquely, therefore, we use just the triplet of parameters $(v, k, \lambda)$ for a BIBD.

For any finite group $H$, the group ring $\mathbb{Z} H$ is defined as the set of all formal sums of elements of $H$, with coefficients from $\mathbb{Z}$. Let $X$ denote a non-empty subset of $H$. The element $\sum_{x \in X} x$ in $\mathbb{Z} H$ is called a simple quantity, and it is denoted as $\underline{X}$. Suppose now that $e \notin X$, where $e$ is the identity element of the group $H$. Then the digraph $\Gamma=\operatorname{Cay}(H, X)$ with vertex set $H$ and dart set $\left\{(x, y): x, y \in H, y x^{-1} \in X\right\}$ is called the Cayley digraph over $H$ with respect to $X$.

\subsection{Strongly Regular Graphs}

A graph $\Gamma$ with adjacency matrix $A=A(\Gamma)$ is called regular, if there exists a positive integer $k$ such that $A J=J A=k J$, where $J$ is the all-one matrix. The number $k$ is called valency of $\Gamma$. A simple regular graph with valency $k$ is said to be strongly regular (SRG, for short) if there exist integers $\lambda$ and $\mu$ such that for each edge $\{u, v\}$ the number of common neighbors of $u$ and $v$ is exactly $\lambda$; while for each non-edge $\{u, v\}$ the number of common neighbors of $u$ and $v$ is equal to $\mu$. Previous condition can be rewritten equivalently into the equation $A^{2}=k I+\lambda A+\mu(J-I-A)$ using the adjacency matrix of $\Gamma$. The quadruple $(n, k, \lambda, \mu)$ is called the parameter set of an SRG $\Gamma$.

\subsection{Directed Strongly Regular Graphs}

A possible generalization of the notion of SRGs for directed graphs was given by Duval [ 5 . While the family of SRGs has been well-studied in the algebraic graph theory cf. [2], the directed version has not received enough attention.

A directed strongly regular graph (DSRG) with parameters $(n, k, t, \lambda, \mu)$ is a regular directed graph on $n$ vertices with valency $k$, such that every vertex is incident with $t$ undirected edges, and the number of paths of length 2 directed from a vertex $x$ to another vertex $y$ is $\lambda$, if there is an arc from $x$ to $y$, and $\mu$ otherwise. In particular, a DSRG with $t=k$ is an SRG, and a DSRG with $t=0$ is a doubly regular tournament. Throughout the paper we consider only DSRGs satisfying $0<t<k$, which are called genuine DSRGs.

The adjacency matrix $A=A(\Gamma)$ of a DSRG with parameters $(n, k, t, \lambda, \mu)$, satisfies $A J=J A=k J$ and $A^{2}=t I+\lambda A+\mu(J-I-A)$.

Example 1. The smallest example of a DSRG is appearing on 6 vertices. Its parameter set is $(6,2,1,0,1)$ and it is depicted in Fig. 1 . 


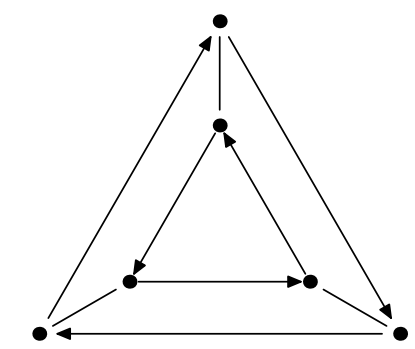

Figure 1: The smallest genuine DSRG.

Remark 1 In this paper, we are using for DSRG's 5-tuple of parameters in the order $(n, k, t, \lambda, \mu)$, however, in several other papers the order $(n, k, \mu, \lambda, t)$ is used.

Proposition 1 ([5]) If $\Gamma$ is a DSRG with parameter set $(n, k, t, \lambda, \mu)$ and adjacency matrix $A$, then the complementary graph $\bar{\Gamma}$ is a DSRG with parameter set $(n, \bar{k}, \bar{t}, \bar{\lambda}, \bar{\mu})$ with adjacency matrix $\bar{A}=J-I-A$, where

$$
\begin{aligned}
\bar{k} & =n-k+1 \\
\bar{t} & =n-2 k+t-1 \\
\bar{\lambda} & =n-2 k+\mu-2 \\
\bar{\mu} & =n-2 k+\lambda .
\end{aligned}
$$

Remark 2 Proposition 11 allows us to restrict our search for the DSRGs with $2 k<n$, due to complementation, and clearly a discovery of a DSRG with new parameter set implies a discovery of a DSRG on the complementary parameter set. As a consequence, throughout the paper we display just the parameter sets satisfying $2 k<n$.

For a directed graph $\Gamma$ let $\Gamma^{T}$ denote the digraph obtained by reversing all the darts in $\Gamma$. Then $\Gamma^{T}$ is called the reverse of $\Gamma$. In other words, if $A$ is the adjacency matrix of $\Gamma$, then $A^{T}$ is the adjacency matrix of $\Gamma^{T}$.

The following proposition was observed by $\mathrm{Ch}$. Pech, and presented in [18]:

Proposition $2([\mathbf{1 8}])$ Let $\Gamma$ be a DSRG. Then the graph $\Gamma^{T}$ is also a DSRG with the same parameter set.

We say that two DSRGs $\Gamma_{1}$ and $\Gamma_{2}$ are equivalent, if $\Gamma_{1} \cong \Gamma_{2}$, or $\Gamma_{1} \cong \Gamma_{2}^{T}$, or $\Gamma_{1} \cong \bar{\Gamma}_{2}$, or $\Gamma_{1} \cong \bar{\Gamma}_{2}^{T}$; otherwise they are called non-equivalent. (In other words, $\Gamma_{1}$ is equivalent to $\Gamma_{2}$ if and only if $\Gamma_{1}$ is isomorphic to $\Gamma_{2}$ or to a graph obtained from $\Gamma_{2}$ via reverse and complementation.) From our point of view the interesting DSRGs are those which are non-equivalent. 
The parameters $n, k, t, \lambda, \mu$ are not independent. Relations to be satisfied for such parameter sets are usually called feasibility conditions. Most important and, in a sense, basic conditions are the following (for their proof see [5]):

$$
k(k+\mu-\lambda)=t+(n-1) \mu .
$$

There exists a positive integer $d$ such that:

$$
\begin{aligned}
d^{2}= & (\mu-\lambda)^{2}+4(t-\mu) \\
d & \mid(2 k-(\mu-\lambda)(n-1)) \\
n-1 & \equiv \frac{2 k-(\mu-\lambda)(n-1)}{d} \quad(\bmod 2) \\
n-1 \geq & \left|\frac{2 k-(\mu-\lambda)(n-1)}{d}\right| .
\end{aligned}
$$

Further:

$$
\begin{aligned}
0 & \leq \lambda<t<k \\
0 & <\mu \leq t<k \\
-2(k-t-1) & \leq \mu-\lambda \leq 2(k-t) .
\end{aligned}
$$

We have to mention that for a feasible parameter set it is not guaranteed that a DSRG with that parameter set does exist. A feasible parameter set for which at least one DSRG $\Gamma$ exists is called realizable, otherwise non-realizable. The smallest example of a non-realizable parameter set is $(14,5,4,1,2)$, what was shown in 18 .

\subsection{Coherent Configurations and Association Schemes}

Under a color graph $\Gamma$ we mean an ordered pair $(V, \mathcal{R})$, where $V$ is a set of vertices and $\mathcal{R}$ a partition of $V \times V$ into binary relations. The elements of $\mathcal{R}$ are called colors, and the number of colors is the rank of $\Gamma$. In other words, a color graph is an edge-colored complete directed graph with loops, whose arcs are colored by the same color if and only if they belong to the same binary relation.

A coherent configuration is a color graph $\mathcal{M}=(\Omega, \mathcal{R}), \mathcal{R}=\left\{R_{i} \mid i \in I\right\}$, such that the following axioms are satisfied:

(i) The diagonal relation $\Delta_{\Omega}=\{(x, x) \mid x \in \Omega\}$ is a union of relations $\cup_{i \in I^{\prime}} R_{i}$, for a suitable subset $I^{\prime} \subseteq I$.

(ii) For each $i \in I$ there exists $i^{\prime} \in I$ such that $R_{i}^{T}=R_{i^{\prime}}$, where $R_{i}^{T}=\{(y, x) \mid(x, y) \in$ $\left.R_{i}\right\}$ is the relation transposed to $R_{i}$.

(iii) For any $i, j, k \in I$, the number $p_{i, j}^{k}$ of elements $z \in \Omega$ such that $(x, z) \in R_{i}$ and $(z, y) \in R_{j}$ is a constant depending only on $i, j, k$, and independent of the choice of $(x, y) \in R_{k}$.

The numbers $p_{i, j}^{k}$ are called intersection numbers, or sometimes structure constants of $\mathcal{M}$. A coherent configuration $\mathcal{M}$ is called commutative, if for all $i, j, k \in I$ we have $p_{i j}^{k}=p_{j i}^{k}$, otherwise non-commutative. 
Let $(G, \Omega)$ be a permutation group. $G$ acts naturally on $\Omega \times \Omega$ by $(x, y)^{g}=\left(x^{g}, y^{g}\right)$. The orbits of this action are called 2-orbits (or orbitals) of $(G, \Omega)$, and denoted by $2-\operatorname{Orb}(G, \Omega)$. It is easy to check that $(\Omega, 2-\operatorname{Orb}(G, \Omega))$ is a coherent configuration for every permutation group $(G, \Omega)$. The coherent configurations which appear in this manner are called Schurian, otherwise non-Schurian.

An association scheme $\mathcal{M}=(\Omega, \mathcal{R})$ is a homogeneous coherent configuration, i.e., where the diagonal relation $\Delta_{\Omega}$ does belong to $\mathcal{R}$. Hence, a very important source of association schemes are transitive permutation groups, since their 2-orbits form a homogeneous coherent configuration.

Let $\mathcal{M}$ be a coherent configuration of rank $r$. To each relation $R_{i}$ in $\mathcal{M}$ we can assign a 0-1-matrix $A_{i}$ such that $\left(A_{i}\right)_{x y}=1 \Longleftrightarrow(x, y) \in R_{i}$. Then clearly $\sum_{i=1}^{r} A_{i}=J$ and $A_{i} A_{j}=\sum_{k=1}^{r} p_{i j}^{k} A_{k}$. Matrices $A_{1}, \ldots, A_{r}$ generate an algebra $\mathcal{W}$ over $\mathbb{C}$, which is called coherent algebra of rank $r$ and degree $n$, and we write $\mathcal{W}=\left\langle A_{1}, \ldots, A_{r}\right\rangle$.

Let $\mathcal{W}_{1}, \mathcal{W}_{2}$ be two coherent algebras of order $n$. Then $\mathcal{W}_{1} \cap \mathcal{W}_{2}$ is again a coherent algebra, therefore, there exists a unique minimal coherent algebra $\mathcal{W}$ containing a given set $\left\{M_{1}, \ldots, M_{t}\right\}$ of $0-1$-matrices of order $n \times n$. This algebra is called coherent closure of $M_{1}, \ldots, M_{t}$ and it is denoted $\left\langle\left\langle M_{1}, \ldots, M_{t}\right\rangle\right\rangle$. In particular, to a DSRG $\Gamma$ with adjacency matrix $A$, we can associate the coherent closure $\mathcal{W}(\Gamma)=\langle\langle A\rangle\rangle$.

To each coherent configuration $\mathcal{M}$, we can assign three groups: $\operatorname{Aut}(\mathcal{M}), \operatorname{CAut}(\mathcal{M})$ and $\operatorname{AAut}(\mathcal{M})$. The (combinatorial) group of automorphisms $\operatorname{Aut}(\mathcal{M})$ consists of the permutations $\phi: \Omega \rightarrow \Omega$ which preserve the relations, i.e., $R_{i}^{\phi}=R_{i}$ for all $R_{i} \in \mathcal{R}$. The color automorphisms preserve relations setwise, i.e., for $\phi: \Omega \rightarrow \Omega$ we have $\phi \in$ $\operatorname{CAut}(\mathcal{M})$ if and only if for all $i \in I$ there exists $j \in I$ such that $R_{i}^{\phi}=R_{j}$. An algebraic automorphism is a bijection $\psi: \mathcal{R} \rightarrow \mathcal{R}$ which satisfies $p_{i j}^{k}=p_{i^{\psi} j^{\psi}{ }^{\psi}}^{k^{4}}$. We refer to [19] for a discussion of these concepts.

Graphs and digraphs can be regarded as binary relations, while association schemes are collections of binary relations in the sense of our definition. Therefore, it is natural to ask:

Question 1. Assume $\mathcal{M}$ is an association scheme of order $n$. Can we obtain a DSRG on $n$ vertices as a union of suitable classes in $\mathcal{M}$ ?

It turns out that there is no standard easy way to reply to the question.

A very important necessary condition posed for the initial association scheme $\mathcal{M}$ was given in [18]:

Theorem 3 ([18]) Let $\Gamma$ be a genuine directed strongly regular graph. Then the coherent closure $\mathcal{W}(\Gamma)$ is non-commutative, and its rank is at least 6.

In other words, we have to consider non-commutative association schemes of rank at least 6 , when we are searching for directed strongly regular graphs as unions of relations in a prescribed association scheme.

Significant part of our results was achieved following the strategy of creating suitable non-commutative association schemes and taking unions of their relations. 


\section{General Approach to the Computer Experimenta- tion}

\subsection{Main Methodology}

Assume that $\mathcal{M}=(\Omega, \mathcal{R})$ is an association scheme and $G=\operatorname{Aut}(\mathcal{M})$. Let $r$ be the rank of $\mathcal{M}$, thus, $\mathcal{M}$ has $r-1$ classes. In many cases below, $G$ acts transitively on $\Omega$. Moreover, $\mathcal{M}$ is the Schurian scheme obtained from permutation group $(G, \Omega)$, however, this restriction is not obligatory in the framework of the described approach.

Let $\Gamma$ be a putative DSRG (with order $n$ ), which is obtained via union of suitable classes of $\mathcal{M}$. Then clearly one has to inspect $2^{r-1}$ possible unions.

First evident restriction is to look simultaneously for all possible parameter sets of DSRGs of order $n$; recall that this data is available at 3 . Typically, in this project, our attention was restricted only to the open parameter sets.

At the second step, one has to consider multisets of valencies of symmetric and antisymmetric classes in $\mathcal{M}$ and to find in advance which subsets of classes of $\mathcal{M}$ may in principle provide a mixed graph with prescribed pair of valencies $(t, k-t)$, respectively. Getting such a list is a simple case of the famous knapsack problem, however, we were using a very naive approach to provide all solutions.

In order to eliminate in further search duplicates of isomorphic graphs it might be helpful to work with the representatives of orbits on sets of relations in the action of the color group $\operatorname{CAut}(\mathcal{M})$. Sometimes, preliminary sorting with the aid of the action of the algebraic group AAut $(\mathcal{M})$ might be also of help. Nevertheless, according to the gathered practical experience, in most of the cases the order of $\operatorname{AAut}(\mathcal{M})$ is relatively small, thus, we are facing exponential complexity in cases of schemes with relatively small valencies. Therefore, we decided to restrict our systematic attempts just to the association schemes of rank not larger than 25 .

Finally, for each selected "suspicious" union of relations from $\mathcal{M}$ we have to check whether it is providing a DSRG or not. Here use of the known structure constants of $\mathcal{M}$ is very crucial: indeed, instead of inspection of the adjacency matrix $A(\Gamma)$ of a putative graph $\Gamma$ we arrange calculations with the tensor of structure constants of $\mathcal{M}$.

The computational scheme outlined above is, in a sense, the ideal plan of activities, which were arranged in the course of computations. In many cases, we preferred to use ingredients of a brute-force approach, rather than to being involved in a more sophisticated programming. Since in many cases it was impossible to execute an exhaustive search, it was substituted by an ad hoc selection of simple "promising" subsets of candidates.

\subsection{Computer Tools}

We run all computations in the software GAP [9] with its share packages GRAPE [28] together with nauty 24] for computation with graphs; an unpublished package COCOII [27] written by S. Reichard for computations with association schemes and coherent configurations; and the package SetOrbit [26] written by Ch. Pech and S. Reichard, and documented in [25], for finding representatives of orbits of group actions on sets of various size. 
In addition, some ad hoc computational tricks were used from time to time, like to exploit a simple variation of the calculation of the coherent closure of an auxiliary graph, which is related to the putative DSRG $\Gamma$, as well as some helpful functions for the calculations with association schemes borrowed from the site [13.

\subsection{Sources for Association Schemes}

Recall that first open parameter set for a DSRG appears for order $n=22$. With growing of $n$ the fraction of open parameter sets is becoming more essential. This dictated our strategy in the selection of candidates for association schemes being considered. In what follows, we report only about successful attempts, resulted in discovery of graphs with open parameter sets. However, as a byproduct, many graphs with known parameter sets were also considered (their comparison with known ones remains as one of tasks for a more systematic approach in the future).

Roughly speaking, we distinguish a few different typical origins in our search:

- use of existing catalogues of association schemes;

- inspection of groups of automorphisms of some "famous" vertex-transitive graphs;

- consideration of incidence structures;

- investigation of Cayley graphs.

In the next section, we are paying reasonable attention to a more detailed discussion of each of these approaches.

\section{Unions of Relations in Association Schemes}

Here we consider several strategies for finding non-commutative association schemes which serve as input for searching new DSRGs.

\subsection{Search Using Catalogue of Small Association Schemes}

For executing our strategy it is enough to consider non-commutative association schemes of small order at the first stage. They are systematically arranged according to their order and rank in the catalogue of Hanaki and Miyamoto [13.

The number of new parameter sets, for which we succeed, using exactly this approach, is 12, see Table 2 in Summary. For several parameter sets, we have found a few nonequivalent DSRGs. Table 1 contains just the digraphs which are mutually non-equivalent. In this Table 1, we display sufficient portion of information for reconstructing discovered DSRGs using the catalogue of association schemes by Hanaki and Miyamoto.

Remark 4 We noticed that in [13] "class" of association schemes is used instead of their "rank". Clearly, the number of classes is less by one than the rank.

From Table 1 it is easy to observe that graph nr. 13 is a (spanning) subgraph of graphs nr. 25 and 26; while nr. 14 is a subgraph of nr. 19. 


\subsection{Actions of Group of Automorphisms of Graphs}

Jørgensen in [17 and [15] announced the existence of a DSRG with the parameter set $(108,10,3,0,1)$. The author provided us the adjacency matrix of this new digraph, and we managed to explain it in terms of unions of relations in the Schurian association scheme of the group of automorphism of the Pappus graph in the action on the ordered triples of its vertices. For more details see [12].

This successful attempt inspired us to go ahead in a similar spirit. In fact, we investigated actions of the group of automorphisms of several symmetric graphs on certain orbits of various $k$-sets and $k$-tuples. Usually, due to high time and space complexity, we took just $k \in\{2,3,4\}$. Restricting group action to an orbit we ensure that the resulted action is transitive on it, and from this action we create the Schurian association scheme. When it passes the test for being non-commutative, then there is sense to execute the search for DSRGs as unions of relations in these schemes. Once more, due to high timecomplexity, we restricted ourselves just for the cases when the rank was not greater than 25 and the size of the orbit not greater than 110. Therefore, our search is far from being exhaustive. If one goes higher with the rank, then he could probably find new DSRGs.

One can find origins of this strategy in [8], Example 3.4. The authors took the lattice graph on 9 points and investigated an action of a subgroup of its group of automorphism on the edges. Our strategy is a slight generalization of it, since we do not consider only the pairs of two adjacent vertices, but also actions on any 2-sets, 3-sets, ordered pairs, ordered triplets of vertices and sometimes on 4-sets, ordered quadruples.

Using our strategy, we succeed in the following cases (the starting "famous" graphs are available via Internet, e.g. from the home page of A. Brouwer):

- from the Petersen graph we obtained a DSRG(60,13, 5, 2,3);

- from the Shrikhande graph we get a DSRG(48, 10, 6, 2,2) and $(48,13,7,2,4)$;

- from the Heawood graph we obtained DSRG $(84,31,17,12,11),(84,29,19,6,12)$ and $(84,39,27,18,18)$, see also Section 4.3 ;

- from the unique $\operatorname{SRG}(21,10,3,6)$ we obtained a $\operatorname{DSRG}(105,36,16,11,13)$;

Explicit descriptions of these digraphs are shown in Appendix.

\subsection{Actions of Group of Automorphisms of Combinatorial De- signs}

Let us now start from a block design $\mathcal{D}=(\mathcal{P}, \mathcal{B})$ with the point set $\mathcal{P}$ and block set $\mathcal{B}$, let $I=I(\mathcal{D})$ be the Levi graph of $\mathcal{D}$, that is the graph with vertex set $\mathcal{P} \cup \mathcal{B}$ and two vertices being adjacent if and only if the corresponding elements of $\mathcal{D}$ are incident. Clearly, $I(\mathcal{D})$ is a bipartite graph. The group $\operatorname{Aut}(I(\mathcal{D})$ ) either coincides with the group $\operatorname{Aut}(\mathcal{D})$, or it is twice larger (the latter corresponds to the case when $\mathcal{D}$ is a symmetric self-dual design).

For a number of designs $\mathcal{D}$, we investigated the action of the group $G=\operatorname{Aut}(I(\mathcal{D}))$ on certain orbits of various $k$-subsets and $k$-tuples of vertices of $I$. The same limitations for values of $k$, order and rank of related Schurian association scheme (like in previous 
section) remain valid. The execution of search for DSRGs has been started, provided the appearing Schurian schemes were non-commutative.

Using this strategy we succeed in the following cases:

- Considering the unique $(7,3,1)$-design is equivalent of consideration of the Heawood graph in the previous section, since the Levi graph of the $(7,3,1)$-design is the Heawood graph;

- from the unique $(9,3,1)$-design we get DSRGs with parameter sets $(72,20,14,4,6)$, $(72,21,15,6,6),(72,22,9,6,7)$;

- from the $(10,4,2)$-design with group of automorphisms of order 720 we get DSRGs with parameter sets $(60,26,20,10,12)$ and $(90,28,16,10,8)$;

- from the $(15,3,1)$-design which has group of automorphisms of order 288 we get DSRG with parameter set $(72,26,10,8,10)$;

- from the $(15,7,3)$-design with group of automorphisms of order 1152 we get a DSRG with parameter set $(72,19,11,2,6)$;

- from the $(25,4,1)$-design with group of automorphisms of order 504 we get a DSRG with parameter set $(63,22,10,7,8)$.

In all these cases, we refer to the description of block designs provided in [4.

Explicit descriptions of all digraphs constructed in this subsection are shown in Appendix.

Example 5 Consider the unique $(7,3,1)$-design $F$, that is the Fano plane. In this case, the group $G$ of order 336 acts transitively on the vertex set of graph $I(F)$ of size 14 . Let us consider configuration, which consists of two lines, their intersection point and another point in one line not belonging to the other line. Clearly, there are $\left(\begin{array}{l}7 \\ 2\end{array}\right) \cdot 4=84$ possibilities to select such a configuration. It is easy to see that both groups $\operatorname{Aut}(F)$ and $G=\operatorname{Aut}(I(F)$ ) (of order 168 and 336, respectively) act transitively on the set $\Omega$ of cardinality 84. The advantage of the group $G$ is that the corresponding association scheme is of rank 25, that is on the edge of our computational possibilities. The remaining details relevant to the precise description of the resulted $D S R G(84,31,17,12,11)$ are in Appendix.

It is worthy to notice that the incidence graph $I(F)$ is isomorphic to the Heawood graph considered above. In fact, the considerations from Heawood graph were fulfilled in advance (this took a few days of computational time) and it was exceptionally extended up to groups of rank 30, which lead to the discovery of $D S R G(84,29,19,6,12)$ and $(84,39,27,18,18)$.

Example 6 Consider the unique $(9,3,1)$-design. We identify its set of points with the set $\mathcal{P}=\{1,2, \ldots, 9\}$ and its set of blocks with

$$
\begin{gathered}
\mathcal{B}=\{\{1,2,3\},\{1,4,7\},\{1,5,9\},\{1,6,8\},\{2,4,9\},\{2,5,8\},\{2,6,7\}, \\
\{3,4,8\},\{3,5,7\},\{3,6,9\},\{4,5,6\},\{7,8,9\}\} .
\end{gathered}
$$


The full group of automorphisms of $(\mathcal{P}, \mathcal{B})$ can be identified with the permutation group $G=\langle(1,7,3,2,6,9,4,5),(4,6,5)(7,8,9)\rangle$ of order 432 and degree 9. (Of course, it could be also regarded as a permutation group of degree $|\mathcal{P}|+|\mathcal{B}|=21$.) Let us consider the action of $G$ on the 3-set $\{1,2,4\}$. There are $72=12 \cdot 3 \cdot 2$ possibilities (from geometric arguments) for such a selection. Denote by $\mathcal{O}$ the entire set of selected configurations. The automorphism group of $(\mathcal{P}, \mathcal{B})$ acts naturally on $\mathcal{O}$ as a permutation group $\tilde{G}$ of degree 72, rank 16. By choosing suitable subsets of 2-orbits of $\tilde{G}$, we get two nonequivalent DSRGs with parameter set $(72,22,9,6,7)$. For a representation of $\tilde{G}$ as a permutation group of degree 72 see the group called $\mathrm{H}_{7}$ in Appendix.

\section{New Sporadic Examples as Cayley Digraphs}

In this section, we construct some new DSRGs of order 32 and 39 as Cayley digraphs. Among them, we obtain the first DSRG with parameter set $(39,16,12,7,6)$.

The following lemma is crucial for testing whether a Cayley digraph is DSRG.

Lemma 7 ([18, [14]) The Cayley digraph $\operatorname{Cay}(G, X)$ is DSRG with parameter set $(v, k, t, \lambda, \mu)$ if and only if the equation $\underline{X} \cdot \underline{X}=t \cdot \underline{e}+\lambda \cdot \underline{X}+\mu \cdot(\underline{G}-\underline{e}-\underline{X})$ holds in $\mathbb{Z} G$.

\subsection{Cayley Digraphs on 32 Vertices}

We now show how to obtain new DSRGs for parameter sets $(32,9,6,1,3),(32,13,9,4,6)$ and $(32,14,10,6,6)$.

Let us take the wreath product group $H=S_{2} \nmid \mathbb{Z}_{4}$ of order 32. (Here for wreath product we follow notation from [22, which is inherited from L.A. Kalužnin.) Each element $h \in H$ can be uniquely represented as $h=\left(g ; k_{1}, k_{2}\right)$, where $g \in S_{2}, k_{1}, k_{2} \in \mathbb{Z}_{4}$. Let $x$ be a generator of $\mathbb{Z}_{4}$, and $\pi=(12) \in S_{2}$. In order to shorten description we display just the triple $i j l$ instead of $\left(\pi^{i} ; x^{j}, x^{l}\right)$.

Let us define six subsets of $H$ :

$$
\begin{aligned}
& X_{1}=\{002,011,012,032,033,100,101,102,103\} \\
& X_{2}=\{010,011,030,031,033,100,102,111,113,121,123,130,132\} \\
& X_{3}=\{010,021,022,023,030,100,102,111,113,121,123,130,132\}, \\
& X_{4}=\{002,011,012,032,033,100,101,102,103,120,121,122,123\} \\
& X_{5}=\{001,003,011,012,032,033,100,101,102,103,110,111,112,113\} \\
& X_{6}=\{001,003,011,012,032,033,100,101,102,103,110,112,131,133\}
\end{aligned}
$$

It is a routine-work to check using Lemma 7 that the following proposition holds:

Proposition 3 The Cayley digraph $\Gamma_{i}=\mathrm{Cay}\left(H, X_{i}\right)$ is a DSRG with parameter set

a) $(32,9,6,1,3)$, for $i=1$;

b) $(32,13,9,4,6)$, for $i=2,3,4$, and 
c) $(32,14,10,6,6)$, for $i=5,6$.

According to [3], DSRGs with parameter sets mentioned in the previous proposition had been constructed in [10, [23].

Using computer algebra system GAP [9] along with the computer package GRAPE [1] in common with nauty 24, we have tested that all the digraphs constructed in Proposition 3 are pairwise non-equivalent and none of these DSRGs were obtained earlier.

\subsection{Cayley Digraphs on 39 Vertices}

In this subsection, we construct DSRGs for all feasible parameter sets on 39 vertices. Hence, we obtain also a DSRG for the parameter set $(39,16,12,7,6)$ for which such a graph has not been known at the time of writing this paper. All these graphs arise as Cayley digraphs over a metacyclic group of order 39. For more constructions from metacyclic groups, we refer the reader to [6].

Let us take the group $G$ presented as

$$
G=\left\langle a, b: a^{13}=b^{3}=e, b a=a^{9} b\right\rangle \leq A G L(1,13)
$$

and let us define eight of its subsets:

$$
\begin{aligned}
& X_{1}=\left\{a, a^{5}, a^{8}, a^{12}, b, a^{2} b, a^{4} b, a^{3} b^{2}, a^{7} b^{2}, a^{11} b^{2}\right\}, \\
& X_{2}=\left\{a, a^{5}, a^{8}, a^{12}, b, a^{4} b, a^{7} b, a^{11} b, a^{2} b^{2}, a^{4} b^{2}, a^{8} b^{2}, a^{11} b^{2}\right\}, \\
& X_{3}=\left\{a, a^{5}, a^{8}, a^{12}, b, a^{4} b, a^{7} b, a^{10} b, a^{3} b^{2}, a^{4} b^{2}, a^{10} b^{2}, a^{11} b^{2}\right\}, \\
& X_{4}=\left\{a^{2}, a^{4}, a^{9}, a^{11}, b, a^{4} b, a^{6} b, a^{11} b, a^{3} b^{2}, a^{5} b^{2}, a^{9} b^{2}, a^{11} b^{2}\right\}, \\
& X_{5}=X_{2} \cup\left\{a^{12} b, a^{3} b^{2}\right\}, \\
& X_{6}=X_{4} \cup\left\{a^{2} b, a^{7} b^{2}\right\} \\
& X_{7}=\left\{a^{2}, a^{4}, a^{9}, a^{11}, a^{2} b, a^{3} b, a^{6} b, a^{8} b, a^{11} b, a^{12} b, b^{2}, a^{3} b^{2}, a^{4} b^{2}, a^{6} b^{2}, a^{7} b^{2}, a^{10} b^{2}\right\}, \\
& X_{8}=\left\{a^{4}, a^{5}, a^{8}, a^{9}, b, a b, a^{4} b, a^{6} b, a^{8} b, a^{10} b, b^{2}, a^{2} b^{2}, a^{4} b^{2}, a^{6} b^{2}, a^{8} b^{2}, a^{10} b^{2}\right\} .
\end{aligned}
$$

Proposition 4 The Cayley digraph $\Gamma_{i}=\operatorname{Cay}\left(G, X_{i}\right)$ is a DSRG with parameter set
a) $(39,10,6,1,3)$, for $i=1$;
b) $(39,12,4,3,4)$, for $i=2,3,4$,
c) $(39,14,6,5,5)$, for $i=5,6$, and
d) $(39,16,12,7,6)$ for $i=7,8$.

Remark 8 The DSRG with parameter set $(39,10,6,1,3)$ is isomorphic to the one constructed in [23] and described using partial sum families. The two digraphs with parameters $(39,16,12,7,6)$ are non-equivalent. 
Remark 9 In [16] the author states that it may happen that the so-called Krein parameter $q_{\theta \theta}^{\theta}$ is always non-negative if 0 and -1 are not eigenvalues of a DSRG $\Gamma$ in consideration. The existence of a DSRG with parameters $(39,10,6,1,3)$ disproves it, since in this case $q_{\theta \theta}^{\theta}=-3 / 8$ (this fact was somehow not mentioned in [23]).

\section{Conclusion and Summary}

The main genre of this paper is computer algebra experimentation for the purposes of algebraic graph theory. Using techniques and ideas, which were before reflected in [6], 8, [7, [18] and [21, the author Š. Gyürki arranged a more systematical search for DSRGs, relying on the above described strategies.

We think that the approaches outlined above carry features of methodological innovations, though in a few cases they simply stem from careful analysis of previous successful computations done by M. Klin et al.

Our next goal was of a definite "sporting" interest: to present examples of new DSRGs for previously open parameter sets. Altogether we reached such a success for 28 new parameter sets, see Table 2 below.

Of course, the foremost goal at a computer algebra experimentation (cf. 20]) is to reach a successful theoretical generalization of the obtained new results. In the case of the sporadic examples of new DSRGs, this would mean to try to embed at least some of the new examples into new infinite classes of DSRGs. We are pleased to claim that this task was successfully fulfilled in the course of our project. In fact, we succeeded to generalize the presented digraph with parameter set $(32,14,10,6,6)$ to the infinite series of DSRGs with parameters $\left(2 n^{2}, 4 n-2,2 n+2, n+2,6\right)$. The corresponding paper is in preparation. Hence, we can finally claim that one more corollary of the reported project is creation of new (striking in the eyes of the authors) patterns of successful insight:

- to observe a short sequence of parameter sets with similar properties;

- to formulate a plausible conjecture about a possible putative infinite series of combinatorial structures;

- to prove this conjecture on purely theoretical level, that is finally, without the use of a computer.

Table 2 below provides a brief summary of our computer aided discoveries.

\section{Acknowledgements}

The first author gratefully acknowledges the contribution of the Scientific Grant Agency of the Slovak Republic under the grant 1/1005/12.

This research was also supported by the Project: Mobility - enhancing research, science and education at the Matej Bel University, ITMS code: 26110230082, under the Operational Program Education cofinanced by the European Social Fund.

We thank L. Jørgensen for generous sharing with us of his preliminary results related to the DSRG on 108 vertices. A long-standing cooperation with Ch. Pech and S. Reichard in the use of computer algebra tools is appreciated. 


\section{References}

[1] Biggs, N.: Algebraic Graph Theory, 2nd ed. Cambridge Mathematical Library, Cambridge University Press, Cambridge (1993) (1st ed. (1974))

[2] Brouwer, A.E., Haemers, W.H.: Spectra of Graphs. Universitext. Springer, New York (2012)

[3] Brouwer, A.E., Hobart, S.: Tables of directed strongly regular graphs (April 2014), http://homepages.cwi.nl/ aeb/

[4] Colbourn, C.J., Dinitz, J.H.: The Handbook of Combinatorial Designs, 2nd ed. Chapman \& Hall/CRC, Boca Raton, FL (2007)

[5] Duval, A.M.: A directed graph version of strongly regular graphs. J. Combin. Th. A $47,71-100(1988)$

[6] Duval, A.M., Iourinski, D.: Semidirect product constructions of directed strongly regular graphs. J. Combin. Th. A 104, 157-167 (2003)

[7] Fiedler, F., Klin, M.H., Muzychuk, M.: Small vertex-transitive directed strongly regular graphs. Discrete Math. 255, 87-115 (2002)

[8] Fiedler, F., Klin, M., Pech, Ch.: Directed strongly regular graphs as elements of coherent algebras. In: Denecke, K., Vogel, H.-J. (eds.) General Algebra and Discrete Mathematics: Proc. Conf. on General Algebra and Discrete Mathematics, Potsdam 1998, pp. 69-87. Shaker Verlag, Aachen (1999)

[9] GAP - Groups, Algorithms, Programming - a System for Computational Discrete Algebra, www.gap-system.org

[10] Godsil, C.D., Hobart, S.A., Martin, W.J.: Representations of directed strongly regular graphs. Europ. J. Combin. 28, 1980-1993 (2007)

[11] Godsil, C.D., Royle, G.: Algebraic Graph Theory. Graduate Texts in Mathematics 207. Springer-Verlag, New York (2001)

[12] Gyürki, Š., Klin, M.: On a new directed strongly regular graph on 108 vertices constructed by Jørgensen, and graphs related to it. Manuscript (2013)

[13] Hanaki, A., Miyamoto, I.: Catalogue of Small Association Schemes. http://kissme.shinshu-u.ac.jp/as/, (accessed: November 2013)

[14] Hobart, S.A., Shaw, T.J.: A note on a family of directed strongly regular graphs. Europ. J. Combin. 20, 819-820 (1999)

[15] Jørgensen, L.K.: New mixed Moore graphs and directed strongly regular graphs. http://vbn.aau.dk/files/166247351/R_2013_13.pdf

[16] Jørgensen, L.K.: Non-existence of directed strongly regular graphs. Discrete Math. 264, 111-126 (2003) 
[17] Jørgensen, L.K.: Variations and generalizations of Moore Graphs, The International Workshop on Optimal Networks Topologies 2012, Bandung. (Slides are available on http://people.math.aau.dk/ leif

[18] Klin, M., Munemasa, A., Muzychuk, M., Zieschang, P.H.: Directed strongly regular graphs obtained from coherent algebras. Lin. Alg. Appl. 377, 83-109 (2004)

[19] Klin, M., Muzychuk, M., Pech, Ch., Woldar, A., Zieschang, P.H.: Association schemes on 28 points as mergings of a half-homogeneous coherent configuration. European J. Combin. 28(7), 1994-2025 (2007)

[20] Klin, M., Pech, Ch., Reichard, S., Woldar, A., Ziv-Av, M.: Examples of computer experimentation in algebraic combinatorics. Ars Math. Contemp. 3(2), 237-258 (2010)

[21] Klin, M., Pech, Ch., Zieschang, P.H.: Flag algebras of block designs: I. Initial notions, Steiner 2-designs and generalized quadrangles. Preprint, MATH-AL-10-1998, Technische Universität Dresden (1998)

[22] Klin, M., Pöschel, R., Rosenbaum, K.: Angewandte Algebra für Mathematiker und Informatiker, Einführung in gruppentheoretisch-kombinatorische Methoden (German). (Applied algebra for mathematicians and information scientists. Introduction to Group-theoretical Combinatorial Methods.) VEB Deutscher Verlag der Wissenschaften. Berlin (1988)

[23] Martinez, L., Araluze, A.: New tools for construction of directed strongly regular digraphs: Difference digraphs and partial sum families. J. Combin. Th. B 100, 720$728(2010)$

[24] McKay, B.D.: nauty user's guide, ver. 1.5, Technical Report TR-CS-90-02. Computer Science Department, Australian National Univ. (1990)

[25] Pech, Ch., Reichard, S.: Enumerating set orbits. in: M. Klin et al., Algorithmic Algebraic Combinatorics and Gröbner Bases, pp. 137-150. Springer, Berlin, Heidelberg (2009)

[26] Pech, Ch., Reichard, S.: The SetOrbit package for GAP. www .math.tu-dresden.de/ pech

[27] Reichard, S.: COCO II, (personal communication)

[28] Soicher, L.H.: GRAPE: A system for computing with graphs and groups, Groups and computation (New Brunswick, 1991), DIMACS Ser. Discrete Math. Theoret. Comput. Sci. 11, pp. 287-291, Amer. Math. Soc., Providence, RI (1993) 


\section{Appendix}

Parameter Sets $(48,10,6,2,2)$ and $(48,13,7,2,4)$

$$
\begin{aligned}
H_{1}= & \langle(1,2,4)(3,11,13,6,7,14)(5,29,22,16,10,26)(8,18,20,9,17,21)(12,24,35) \\
& (15,33,41,37,28,46)(19,32,44)(23,25,36,40,27,38)(30,42,43)(31,34,45,47,39,48), \\
& (1,3)(2,5)(4,8)(6,12)(7,15)(9,19)(10,23)(11,18)(13,25)(14,26)(16,30) \\
& (17,31)(20,33)(22,34)(24,36)(27,39)(32,45)(35,46)(37,47)(38,44)(41,42)(43,48)\rangle
\end{aligned}
$$

Digraph with vertex set $\{1,2, \ldots, 48\}$ and dart set $(1,3)^{H_{1}} \cup(1,5)^{H_{1}} \cup(1,15)^{H_{1}}$ is a DSRG $(48,10,6,2,2)$, while the one with dart set $(1,2)^{H_{1}} \cup(1,5)^{H_{1}} \cup(1,12)^{H_{1}} \cup(1,19)^{H_{1}} \cup$ $(1,23)^{H_{1}}$ is a DSRG $(48,13,7,2,4)$.

Parameter Set $(60,13,5,2,3)$

$$
\begin{aligned}
H_{2}= & \langle(1,5,16,40,21)(2,8,24,14,37)(3,10,29,28,9)(4,12,26,39,15) \\
& (6,18,44,33,54)(7,20,36,49,23)(11,31,53,58,60)(13,35,34,42,32) \\
& (17,41,27,50,43)(19,45,51,57,25)(22,46,55,56,48)(30,52,38,59,47), \\
& (1,23,40,3,43,34)(2,15,32,6,28,12)(4,37,22,11,57,8)(5,35,46,14,41,58) \\
& (7,21,49,19,47,56)(9,54,30,25,60,17)(10,16,53,27,24,51) \\
& (13,39,52,33,20,55)(18,42,50,31,48,59)(26,29,45,36,44,38)\rangle .
\end{aligned}
$$

Digraph with vertex set $\{1,2, \ldots, 60\}$ and dart set $(1,5)^{H_{2}} \cup(1,6)^{H_{2}} \cup(1,7)^{H_{2}} \cup(1,8)^{H_{2}} \cup$ $(1,12)^{H_{2}} \cup(1,19)^{H_{2}} \cup(1,20)^{H_{2}} \cup(1,49)^{H_{2}}$ is a DSRG with parameter set $(60,13,5,2,3)$.

Parameter Set $(60,26,20,10,12)$

$$
\begin{aligned}
H_{3}= & \langle(1,23,54,6,49,40)(2,32,50,14,57,37)(3,31,27,12,45,43)(4,16,41,9,30,28) \\
& (5,22,46,24,26,53)(7,11,58,21,39,47)(8,10,36,17,19,60)(13,15,20,33,35,52) \\
& (18,48,59,44,38,42)(25,29,51,34,56,55), \\
& (1,56,31,34,16,55)(2,48,15,18,32,42)(3,24,60,46,41,47)(4,38,10,44,23,59) \\
& (5,33,53,20,58,37)(6,39,45,21,30,26)(7,29,22,25,11,51)(8,17,43,27,54,40) \\
& (9,57,19,14,49,35)(12,52,36,50,28,13)\rangle .
\end{aligned}
$$

Digraphs with vertex set $\{1,2, \ldots, 60\}$ and dart set $(1,3)^{H_{3}} \cup(1,5)^{H_{3}} \cup(1,12)^{H_{3}} \cup$ $(1,17)^{H_{3}} \cup(1,36)^{H_{3}},(1,12)^{H_{3}} \cup(1,17)^{H_{3}} \cup(1,23)^{H_{3}} \cup(1,36)^{H_{3}}$ are two non-equivalent DSRGs with parameter set $(60,26,20,10,12)$.

Parameter Set $(63,22,10,7,8)$

$$
\begin{aligned}
H_{4}= & \langle(1,3,8,19,38,33,61)(2,5,12,7,17,34,20,29,50,16,32,25,45,62) \\
& (4,9,21,11,18,36,6,15,30,24,42,55,13,27)(10,23,41,26,35,40,28,48,63,53,37,56,54,44) \\
& (14,39,57,52,43,31,22)(46,47,59,49,60,51,58) \\
& (1,2)(3,6)(5,10,50,61)(7,8)(9,14)(11,24,42,51)(12,19)(16,35)(17,46) \\
& (18,31)(20,30)(21,58)(23,39,63,29)(25,55)(26,32)(27,41)(28,33)(36,47)(37,43)(48,57) \\
& (52,56)(53,59)(60,62) .
\end{aligned}
$$

Digraph with vertex set $\{1,2, \ldots, 63\}$ and dart set $(1,2)^{H_{4}} \cup(1,3)^{H_{4}} \cup(1,5)^{H_{4}}$ is a DSRG with parameter set $(63,22,10,7,8)$. 
Parameter Set $(72,19,11,2,6)$

$$
\begin{aligned}
H_{5}= & \langle(1,6,47,35,8,23,56,24)(2,13,36,32,4,40,64,41)(3,10,61,46,19,15,54,16) \\
& (5,18,17,65,12,26,30,57)(7,21,51,44,11,28,63,29)(9,31,25,72,20,43,42,68) \\
& (14,53,49,66,27,50,33,59)(22,38,60,71,39,34,45,70)(48,52)(55,58,69,67), \\
& (1,28,42,37,70,44,8,10,45,55,72,41)(2,15,30,52,71,46,4,21,33,67,68,24) \\
& (3,40,25,48,59,32,19,6,60,58,65,29)(5,34,64,12,38,63,20,53,54,9,50,56) \\
& (7,23,17,62,66,35,11,13,49,69,57,16)(14,26,36,22,43,47,39,31,61,27,18,51), \\
& (1,15,51,72,48,12)(2,28,61,65,37,5)(3,23,36,68,62,20)(4,13,64,59,69,39) \\
& (6,56,70,67,22,8)(7,40,47,57,52,9)(10,54,71,55,14,19)(11,21,63,66,58,27) \\
& (16,50,60,35,18,30)(17,24,34,49,32,26)(25,41,38,33,44,43)(29,53,45,46,31,42)\rangle .
\end{aligned}
$$

Digraph with vertex set $\{1,2, \ldots, 72\}$ and dart set $(1,2)^{H_{5}} \cup(1,7)^{H_{5}} \cup(1,10)^{H_{5}} \cup(1,42)^{H_{5}}$ is a DSRG with parameter set $(72,19,11,2,6)$.

Parameter Sets $(72,20,14,4,6)$ and $(72,21,15,6,6)$

$$
\begin{aligned}
H_{6}= & \langle(1,61,53,14,31,33,63,48)(2,41,26,16,59,42,51,20)(3,71,28,21,34,38,49,13) \\
& (4,39,44,40,35,6,52,36)(5,64,11,9,69,22,50,30)(7,25,60,68,17,10,58,23) \\
& (8,72,19,32,47,29,56,27)(12,43,70,57,24,18,67,15)(37,45,65,55,62,46,54,66), \\
& (2,18,3)(4,24,37)(5,10,8)(7,27,45)(9,20,54)(11,33,25)(12,13,46)(14,17,62) \\
& (15,29,31)(16,30,65)(19,22,34)(21,48,55)(23,38,39)(26,44,43)(28,42,47)(32,35,66) \\
& (36,70,59)(40,51,56)(41,58,61)(49,63,50)(52,64,67)(53,60,69)\rangle,
\end{aligned}
$$

Digraph with vertex set $\{1,2, \ldots, 72\}$ and dart set $(1,7)^{H_{6}} \cup(1,21)^{H_{6}} \cup(1,40)^{H_{6}} \cup(1,57)^{H_{6}}$ is a DSRG with parameter set $(72,20,14,4,6)$.

Digraph with vertex set $\{1,2, \ldots, 72\}$ and dart set $(1,2)^{H_{6}} \cup(1,4)^{H_{6}} \cup(1,15)^{H_{6}} \cup$ $(1,36)^{H_{6}}$ is a DSRG with parameter set $(72,21,15,6,6)$.

Parameter Set $(72,22,9,6,7)$

$$
\begin{aligned}
H_{7}= & \langle(1,44,55,3,20,25,48,28)(2,36,64,42,19,51,69,49)(4,37,9,34,13,39,17,38) \\
& (5,53,47,68,14,26,66,71)(6,18,59,10,12,31,40,16)(7,46,29,57,8,56,70,62) \\
& (11,72,67,15,58,23,45,54)(21,41,30,50,61,43,33,27)(22,35,65,24,52,60,32,63), \\
& (1,32,51)(2,43,53)(3,45,30)(4,69,31)(5,52,19)(6,33,60)(7,61,20)(8,16,72) \\
& (9,48,66)(10,68,65)(11,56,26)(12,15,50)(13,17,70)(14,49,58)(18,47,25)(21,71,37) \\
& (22,62,46)(23,39,36)(24,34,35)(27,67,38)(28,63,54)(29,40,44)(41,42,57)(55,59,64)\rangle,
\end{aligned}
$$

Digraphs with vertex set $\{1,2, \ldots, 72\}$ and dart set $(1,2)^{H_{7}} \cup(1,10)^{H_{7}} \cup(1,14)^{H_{7}} \cup$ $(1,24)^{H_{7}} \cup(1,71)^{H_{7}}$, and $(1,2)^{H_{7}} \cup(1,10)^{H_{7}} \cup(1,24)^{H_{7}} \cup(1,45)^{H_{7}} \cup(1,72)^{H_{7}}$ are two non-equivalent DSRGs with parameter set $(72,22,9,6,7)$. 
Parameter Set $(72,26,10,8,10)$

$$
\begin{aligned}
H_{8}= & \langle(1,10,9,12,25,18)(2,49,50,46,41,59)(3,63,11,47,20,69)(4,31,33,60,52,66) \\
& (5,56,71,51,38,53)(6,36,17)(7,35,57,19,27,39)(8,24,54,61,14,40)(15,45,68) \\
& (13,26,43,28,67,58)(16,23,55)(21,65,30,48,37,32)(22,44,42,34,62,72)(29,64,70), \\
& (1,58)(2,67)(3,26)(4,68)(5,45)(6,72)(7,13)(8,15)(9,53)(10,59)(11,28) \\
& (12,70)(14,49)(16,43)(17,30)(18,35)(19,38)(20,51)(21,55)(22,65)(23,44) \\
& (24,66)(25,56)(27,47)(29,54)(31,48)(32,60)(33,39)(34,57)(36,63)(37,41) \\
& (40,62)(42,61)(46,71)(50,69)(52,64) \\
& (1,63,36,58,5,45)(2,40,18,59,48,13)(3,54,6,56,21,68)(4,55,25,72,29,26) \\
& (7,31,10,35,62,67)(8,23,9,30,64,28)(11,52,17,53,44,15)(12,70,51,43,16,20) \\
& (14,57,22,37,39,66)(19,42,69,27,60,71)(24,33,41,65,34,49)(32,47,50,61,38,46)\rangle .
\end{aligned}
$$

Digraph with vertex set $\{1,2, \ldots, 72\}$ and dart set $(1,3)^{H_{8}} \cup(1,14)^{H_{8}} \cup(1,19)^{H_{8}} \cup$ $(1,20)^{H_{8}} \cup(1,58)^{H_{8}}$ is a DSRG with parameter set $(72,26,10,8,10)$.

Parameter Sets $(84,29,19,6,12),(84,31,17,12,11)$ and $(84,39,27,18,18)$

$$
\begin{aligned}
H_{9}= & \langle(1,42,52,14,67,78,38,39)(2,28,54,40,18,37,41,23)(3,64,77,82,80,53,33,56) \\
& (4,34,30,15,79,83,61,58)(5,63,74,27,32,72,68,31)(6,13,36,12) \\
& (7,11,25,29,10,35,43,45)(8,51,75,69,50,55,26,49)(9,20,57,84,81,70,62,22) \\
& (16,24,46,76,71,73,17,21)(19,44,59,48,47,60,65,66) \\
& (1,71,8)(2,72,20)(3,10,60)(4,68,23)(5,82,14)(6,63,52)(7,75,24)(9,25,28) \\
& (11,16,54)(12,44,74)(13,42,49)(15,67,77)(17,73,39)(18,78,30)(19,81,27) \\
& (21,38,40)(22,65,29)(26,59,36)(31,58,80)(32,84,57)(33,69,45)(34,41,37) \\
& (35,56,43)(46,51,76)(47,79,64)(48,55,83)(50,53,61)(62,70,66)\rangle .
\end{aligned}
$$

Digraph with dart set $(1,3)^{H_{9}} \cup(1,5)^{H_{9}} \cup(1,6)^{H_{9}} \cup(1,7)^{H_{9}} \cup(1,8)^{H_{9}} \cup(1,9)^{H_{9}} \cup$ $(1,19)^{H_{9}} \cup(1,26)^{H_{9}} \cup(1,29)^{H_{9}} \cup(1,40)^{H_{9}}$ is a $\operatorname{DSRG}(84,29,19,6,12)$, digraph with dart set $(1,2)^{H_{9}} \cup(1,3)^{H_{9}} \cup(1,5)^{H_{9}} \cup(1,7)^{H_{9}} \cup(1,19)^{H_{9}} \cup(1,20)^{H_{9}} \cup(1,27)^{H_{9}} \cup(1,30)^{H_{9}} \cup$ $(1,31)^{H_{9}} \cup(1,35)^{H_{9}}$ is a $\operatorname{DSRG}(84,31,17,12,11)$, digraphs with dart set $(1,3)^{H_{9}} \cup(1,4)^{H_{9}} \cup$ $(1,5)^{H_{9}} \cup(1,6)^{H_{9}} \cup(1,13)^{H_{9}} \cup(1,14)^{H_{9}} \cup(1,19)^{H_{9}} \cup(1,26)^{H_{9}} \cup(1,29)^{H_{9}} \cup(1,30)^{H_{9}} \cup$ $(1,35)^{H_{9}} \cup(1,40)^{H_{9}} \cup(1,46)^{H_{9}}$ and $(1,3)^{H_{9}} \cup(1,4)^{H_{9}} \cup(1,5)^{H_{9}} \cup(1,6)^{H_{9}} \cup(1,13)^{H_{9}} \cup$ $(1,15)^{H_{9}} \cup(1,19)^{H_{9}} \cup(1,21)^{H_{9}} \cup(1,23)^{H_{9}} \cup(1,26)^{H_{9}} \cup(1,29)^{H_{9}} \cup(1,30)^{H_{9}} \cup(1,40)^{H_{9}}$ are $\operatorname{DSRG}(84,39,27,18,18)$.

Parameter Set $(90,28,16,10,8)$

$$
\begin{aligned}
H_{10}= & \langle(1,52,41,43,40)(2,15,79,34,24)(3,75,64,66,56)(4,63,60,14,31)(5,86,69,76,36) \\
& (6,20,13,82,83)(7,25,73,48,12)(8,39,88,37,45)(9,33,32,62,74)(10,70,51,59,17) \\
& (11,80,53,65,67)(16,61,58,90,55)(18,26,72,21,27)(19,81,28,42,49) \\
& (22,71,78,54,68)(23,47,46,85,44)(29,50,38,87,77)(30,84,57,89,35) \\
& (1,79,84,2,60,78)(3,73,61,7,83,77)(4,41,53)(5,34,50,10,48,68) \\
& (6,70,47,12,49,38)(8,51,23,15,72,36)(9,25,88,17,18,69)(11,26,75,20,40,55) \\
& (13,64,28)(14,86,33,24,67,22)(16,82,80,27,74,85)(19,39,52,31,56,35)(21,32,58) \\
& (29,43,71,42,59,89)(30,63,81,45,44,62)(37,46,57)(54,66,87,65,76,90)\rangle .
\end{aligned}
$$


Digraph with vertex set $\{1,2, \ldots, 90\}$ and dart set $(1,2)^{H_{10}} \cup(1,5)^{H_{10}} \cup(1,15)^{H_{10}} \cup$ $(1,20)^{H_{10}} \cup(1,40)^{H_{10}}$ is a DSRG with parameter set $(90,28,16,10,8)$.

Parameter Set $(105,36,16,11,13)$

$$
\begin{aligned}
H_{11}= & \langle(1,96,45,103,3,68,12,105,32,79)(2,82,50,100,10,42,15,102,53,60)(13,25,77,97,49) \\
& (4,47,28,104,36,56,5,85,64,98)(6,99,22,93,7,87,20,101,11,90)(16,39,88,83,44) \\
& (8,91,38,84,17,69,24,95,29,74)(9,73,40,92,14,70,18,94,26,81)(21,72,89,65,27) \\
& (19,55,57,86,43,41,30,78,71,67)(23,34,61,75,48)(31,46,62,80,37) \\
& (33,35,66,76,63)(51,54,52,58,59) \\
& (1,97)(2,89)(3,88)(4,77)(5,83)(6,76)(7,80)(8,62)(9,63)(10,72)(11,64)(12,65) \\
& (13,66)(14,50)(15,49)(16,52)(17,59)(18,58)(19,104)(20,53)(21,51)(22,54)(23,102) \\
& (24,36)(25,37)(26,35)(27,46)(28,44)(29,45)(30,100)(31,38)(32,40)(33,39)(34,98) \\
& (41,105)(42,95)(43,94)(47,92)(48,91)(55,103)(56,85)(57,84)(60,82)(61,81)(67,99) \\
& (68,101)(69,73)(70,74)(71,75)(78,93)(79,96)\rangle
\end{aligned}
$$

Digraph with vertex set $\{1, \ldots, 105\}$ and dart set $(1,2)^{H_{11}} \cup(1,4)^{H_{11}} \cup(1,10)^{H_{11}} \cup$ $(1,22)^{H_{11}} \cup(1,26)^{H_{11}}$ is a DSRG with parameter set $(105,36,16,11,13)$. 
Table 1: DSRGs from small association schemes (Used abbreviations: AS - association scheme, nr.cat - number of the AS in the catalogue)

\begin{tabular}{|r|l|l|c|c|c|}
\hline nr. & $(n, k, t, \lambda, \mu)$ & Union of relations & AS order & AS rank & nr.cat \\
\hline 1 & $(30,13,11,6,5)$ & $1,2,4,6,8$ & 30 & 11 & 184 \\
2 & $(36,13,7,4,5)$ & $4,5,6,9$ & 36 & 11 & 49 \\
3 & $(36,13,7,4,5)$ & $1,2,3,4,8,12,14,16$ & 36 & 20 & 28 \\
4 & $(36,13,7,4,5)$ & $1,2,3,4,8,12,14,17$ & 36 & 20 & 28 \\
5 & $(36,13,7,4,5)$ & $1,2,3,4,5,10,13,14$ & 36 & 20 & 30 \\
6 & $(36,13,7,4,5)$ & $1,2,3,4,6,8,12,18$ & 36 & 20 & 40 \\
7 & $(36,13,7,4,5)$ & $1,2,3,4,6,8,13,16$ & 36 & 20 & 40 \\
8 & $(36,13,11,2,6)$ & $1,3,5,7,10$ & 36 & 13 & 57 \\
9 & $(45,16,8,5,6)$ & $1,3,5,8$ & 45 & 10 & 18 \\
10 & $(45,16,8,5,6)$ & $2,3,5,8$ & 45 & 10 & 18 \\
11 & $(50,16,10,3,6)$ & $1,2,6,9,13$ & 50 & 14 & 9 \\
12 & $(50,23,13,10,11)$ & $1,2,6,8,10,12$ & 50 & 14 & 17 \\
13 & $(54,8,3,2,1)$ & $1,6,8,12$ & 54 & 18 & 103 \\
14 & $(54,16,12,6,4)$ & $4,5,10,12,16$ & 54 & 18 & 109 \\
15 & $(54,19,9,6,7)$ & $1,3,4,6,11,14,16$ & 54 & 18 & 111 \\
16 & $(54,19,9,6,7)$ & $1,3,4,6,11,14,17$ & 54 & 18 & 111 \\
17 & $(54,20,16,6,8)$ & $2,3,4,5,11,13,14$ & 54 & 18 & 109 \\
18 & $(54,20,16,6,8)$ & $8,9,11,13,14$ & 54 & 18 & 109 \\
19 & $(54,21,17,8,8)$ & $1,2,3,4,5,10,12,16$ & 54 & 18 & 109 \\
20 & $(54,21,17,8,8)$ & $1,8,9,10,12,16$ & 54 & 18 & 109 \\
21 & $(54,25,14,11,12)$ & $1,2,4,6,10,12,14,16$ & 54 & 18 & 106 \\
22 & $(54,25,14,11,12)$ & $1,2,4,6,10,12,15,17$ & 54 & 18 & 106 \\
23 & $(54,25,14,11,12)$ & $1,2,4,6,10,13,15,16$ & 54 & 18 & 106 \\
24 & $(54,25,14,11,12)$ & $1,2,4,6,11,13,15,17$ & 54 & 18 & 106 \\
25 & $(54,25,14,11,12)$ & $1,2,6,8,10,12,14,16$ & 54 & 18 & 103 \\
26 & $(54,25,14,11,12)$ & $1,2,6,8,10,12,15,17$ & 54 & 18 & 103 \\
27 & $(54,25,14,11,12)$ & $1,2,6,8,10,13,14,17$ & 54 & 18 & 103 \\
28 & $(54,25,14,11,12)$ & $1,2,6,8,11,13,14,16$ & 54 & 18 & 103 \\
\hline
\end{tabular}


Table 2: Summary. (Used abbreviations: ps - Is parameter set new?; am - The amount of new constructed DSRGs.)

\begin{tabular}{|c|c|c|c||c|c|c|c|}
\hline$(n, k, t, \lambda, \mu)$ & ps & am & constructed in & $(n, k, t, \lambda, \mu)$ & ps & am & constructed in \\
\hline$(30,13,11,6,5)$ & Yes & 1 & Section 4.1 & $(54,19,9,6,7)$ & Yes & 2 & Section 4.1 \\
$(32,9,6,1,3)$ & No & 1 & Section 5.1 & $(54,20,16,6,8)$ & Yes & 2 & Section 4.1 \\
$(32,13,9,4,6)$ & No & 3 & Section 5.1 & $(54,21,17,8,8)$ & Yes & 2 & Section 4.1 \\
$(32,14,10,6,6)$ & No & 2 & Section 5.1 & $(54,25,14,11,12)$ & Yes & 8 & Section 4.1 \\
$(36,13,7,4,5)$ & Yes & 6 & Section 4.1 & $(60,13,5,2,3)$ & Yes & 1 & Section 4.2 \\
$(36,13,11,2,6)$ & Yes & 1 & Section 4.1 & $(60,26,20,10,12)$ & Yes & 2 & Section 4.3 \\
$(39,10,6,1,3)$ & No & 0 & Section 5.2 & $(63,22,10,7,8)$ & Yes & 1 & Section 4.3 \\
$(39,12,4,3,4)$ & No & 3 & Section 5.2 & $(72,19,11,2,6)$ & Yes & 1 & Section 4.3 \\
$(39,14,6,5,5)$ & No & 2 & Section 5.2 & $(72,20,14,4,6)$ & Yes & 1 & Section 4.3 \\
$(39,16,12,7,6)$ & Yes & 2 & Section 5.2 & $(72,21,15,6,6)$ & Yes & 1 & Section 4.3 \\
$(45,16,8,5,6)$ & Yes & 2 & Section 4.1 & $(72,22,9,6,7)$ & Yes & 2 & Section 4.3 \\
$(48,10,6,2,2)$ & Yes & 1 & Section 4.2 & $(72,26,10,8,10)$ & Yes & 1 & Section 4.3 \\
$(48,13,7,2,4)$ & Yes & 1 & Section 4.2 & $(84,29,19,6,12)$ & Yes & 1 & Section 4.2 \\
$(50,16,10,3,6)$ & Yes & 1 & Section 4.1 & $(84,31,17,12,11)$ & Yes & 1 & Section 4.2 \\
$(50,23,13,10,11)$ & Yes & 1 & Section 4.1 & $(84,39,27,18,18)$ & Yes & 2 & Section 4.2 \\
$(54,8,3,2,1)$ & Yes & 1 & Section 4.1 & $(90,28,16,10,8)$ & Yes & 1 & Section 4.3 \\
$(54,16,12,6,4)$ & Yes & 1 & Section 4.1 & $(105,36,16,11,13)$ & Yes & 1 & Section 4.2 \\
\hline
\end{tabular}

